\title{
ESTILO PERSONAL DEL TERAPEUTA DE PSICÓLOGOS QUE TRABAJAN EN AUTISMO
}

\section{PERSONAL STYLE OF THE THERAPIST OF PSYCHOLOGIST WHO WORK WITH AUTISM}

\author{
Leandro Casari ${ }^{1}$, Fernanda Assennato ${ }^{2}$, Sofía Grzona ${ }^{2}$ \\ 1: INCIHUSA - CONICET CCT Mendoza, Argentina. Pontificia Universidad Católica Argentina \\ 2: Facultad de Psicología, Universidad del Aconcagua, Mendoza, Argentina.
}

Cómo referenciar este artículo/How to reference this article:

Casari, L., Assennato, F. y Grzona, S. (2017). Estilo personal del terapeuta de psicólogos que trabajan en autismo. Revista de Psicoterapia, 28(108), 169-188.

\begin{abstract}
Resumen
El Estilo Personal del Terapeuta (EPT) es un constructo con múltiples dimensiones que se encuentra en un proceso terapéutico, y detalla diferentes funciones, las cuales expresan disposiciones, rasgos y actitudes que el terapeuta muestra en su ejercicio profesional. Los objetivos de la investigación fueron: analizar si existen diferencias en el Estilo Personal del Terapeuta entre los Psicólogos que trabajan con Trastornos del Espectro Autista y aquellos que se dedican a la Psicología Clínica en general, y examinar el efecto del enfoque teórico sobre el EPT en cada grupo de trabajo. La muestra de este estudio estuvo conformada por 64 psicoterapeutas divididos en dos grupos: 32 dedicados al trabajo en TEA y los restantes a la clínica en general. Como instrumento se aplicó el Cuestionario de Estilo Personal del Terapeuta. A nivel de resultados se obtuvieron diferencias significativas en la función de Involucración $(\mathrm{p}<.05)$, donde los terapeutas de niños con TEA obtuvieron puntajes superiores. Por otro lado, el enfoque teórico manifestó resultados significativos sobre el EPT en ambos grupos de terapeutas, en las funciones Atencional y Expresiva, y exclusivamente en los terapeutas de autismo en la función Operativa.

Palabras clave: estilo personal del terapeuta, psicoterapia, trastornos del espectro autista, psicoterapeutas.
\end{abstract}

\begin{abstract}
The Personal Therapist Style (PST) is a multi-dimensional construct present in the therapeutic process, and details distinct functions, which express dispositions, traits and attitudes that the therapist shows in his professional practice. The objectives of the research were: to analyze if there are differences in the PST between psychologists working with Autistic Spectrum Disorders (ASD) and those working in General Clinical Psychology, and to examine the effect of the theoretical approach on PST in each workgroup. The sample of this study was made up of 64 psychotherapists divided into two groups: 32 dedicated to work in ASD and the remainder to the clinic in general. The Therapist Personal Style Questionnaire was used as instrument. As a result, there were significant differences in the Engagement function $(\mathrm{p}<.05)$, where the therapists of children with ASD obtained higher scores. On the other hand, the theoretical approach showed meaningful results on PST in both groups of therapists, in the Attentional and Expressive functions, and exclusively in autism therapists in the Operative function.

Keywords: personal style of the therapist, psychotherapy, autism spectrum disorders, psychotherapist.
\end{abstract}

Fecha de recepción: 10-7-2017. Fecha de aceptación v1: 3-9-2017. Fecha de aceptación v2: 28-9-2017.

Correspondencia sobre este artículo:

E-mail: Imcasari@mendoza-conicet.gov.ar

Dirección postal: INCIHUSA - CONICET CCT Mendoza, Argentina. Pontificia Universidad Católica Argentina

(C) 2017 Revista de Psicoterapia 
El Estilo Personal del Terapeuta (en adelante EPT) surge como constructo en el año 1998, donde se lo define del siguiente modo:

(...) un conjunto de condiciones singulares que llevan a un terapeuta a trabajar en un modo particular en su trabajo. Se refiere a las características normales que cada terapeuta imprime a su trabajo como resultado de su particular manera de ser, más allá del foco de su trabajo y de los requerimientos específicos de la tarea (Fernández-Álvarez, García y Schreb, 1998, p. 352, la traducción nos pertenece).

El constructo se compone de cinco funciones que interactúan a lo largo del proceso terapéutico variando su peso relativo y se expresan como una unidad. También se aclara que estas funciones no cubren todas las acciones que provienen del terapeuta, pero son altamente representativas. Las mismas son bipolares, ya que se expresan en un continuum de valores extremos y los terapeutas generalmente se ubicarían en un punto específico de cada dimensión (Fernández-Álvarez, García, Lo Bianco y Corbella, 2003).

De acuerdo con los autores, las funciones del EPT son cinco: Instruccional, Atencional, Operativa, Expresiva e Involucración.

La función Instruccional está relacionada a la manera de estructurar el encuadre terapéutico, donde los polos son representados por actitudes de flexibilidad o rigidez. Incluye las reglas acerca de las tareas que se realizarán durante las sesiones, como también a lo largo de la terapia, como por ejemplo horarios, honorarios, lugar, y todos los aspectos relacionados al contrato terapéutico (Fernández-Álvarez et al., 2003). Los hallazgos de las investigaciones en el EPT muestran que la función Instruccional estaría vinculada a la alianza terapéutica (Corbella et al., 2007), a variables de personalidad del terapeuta tales como la teoría de la mente o su patrón de apego (Corbella et al. 2009 a), y también a factores profesionales del terapeuta como la duración del tratamiento predominante o enfoque teórico (Vázquez y Gutiérrez, 2015). Sin embargo, es la función del EPT que más inconvenientes ha arrojado en su operacionalización en diversos estudios psicométricos (Silva y Guedes, 2016).

La función Atencional abarca los modos en que el terapeuta busca información terapéutica, donde las acciones básicas son escuchar y preguntar (Fernández Álvarez et al., 2003). Las posibles polaridades son: atención abierta, que implica una actitud pasiva y receptiva, con menor cantidad de intervenciones para recopilar información (Fernández-Álvarez y García, 1998); y, atención focalizada, donde los terapeutas toman una postura más activa y específica en su búsqueda de información. A nivel de resultados, la función Atencional ha mostrado estar vinculada a variables profesionales del terapeuta, tales como experiencia, entrenamiento, enfoque teórico, concepción epistemológica y duración predominante de los tratamientos (Casari, Albanesi y Maristany, 2013; Grzona, Muñoz, Casari y Videla, 2016; Lee, Neimeyer y Rice, 2013; Vázquez y Gutiérrez, 2015), también se ha relacionado con variables de personalidad de los mismos, como por ejemplo el 
estilo de apego y dirección de intereses (Genise, 2015; Genise y García, 2016), y con aspectos demográficos tales como el sexo y la edad (Castañeiras, Ledesma, García y Fernández-Álvarez, 2008). También se reportaron hallazgos acerca de su relación con la alianza terapéutica (Hermosa-Bossano, 2010).

La función Operativa, también llamada de Implementación Instrumental, es definida como "las acciones directamente conectadas con las intervenciones terapéuticas específicas" (Fernández Álvarez et al., 2003, p. 119, la traducción nos pertenece). Aquí los terapeutas pueden optar por un rol directivo en sus abordajes, señalando e indicando los caminos a seguir (como en los manuales de tratamiento), o bien, utilizan un modo opuesto caracterizado por ser flexible y con mayor participación del paciente en el proceso, más común en abordajes que apuntan a la personalidad global del paciente (las polaridades de la función Operativa son: pautado - espontáneo). La función Operativa es sensible para percibir diferencias entre terapeutas según las siguientes variables: enfoques teóricos (Casari et al., 2013), años de experiencia (Castañeiras, García, Lo Bianco y Fernández-Álvarez, 2006), inclinación constructivista o racionalista de trabajo (Lee et al., 2013), personalidad (Genise, 2015) y edad (Castañeiras et al., 2008). También posee relaciones con la alianza terapéutica al interactuar con la resistencia del paciente (Corbella et al., 2007).

La función Expresiva puede ser entendida como las "acciones realizadas por el terapeuta para asegurar la comunicación emocional con el paciente. Es uno de los aspectos centrales de la empatía." (Fernández Álvarez et al., 2003, p. 118, la traducción nos pertenece). También puede considerarse la manera afectiva en que el terapeuta se relaciona con sus pacientes. Esta función explora la distancia comunicacional que cada profesional tiende a establecer con su paciente y se refleja en la intensidad de los intercambios emocionales que éste fomenta, o en el nivel de tolerancia hacia la exposición de sus propias emociones. Las polaridades de la misma oscilan entre baja y alta proximidad emocional. Como variable, ha demostrado relaciones significativas con: enfoque teórico (Casari et al., 2013), experiencia profesional (Castañeiras et al., 2006), concepción epistemológica (Lee et al., 2013) y personalidad (Genise, 2015). Así como también, se encontraron vínculos con la alianza terapéutica (Moya, 2015).

La función de Involucración es definida como "el conjunto de conductas explícitas e implícitas conectadas con el compromiso del terapeuta con su tarea en general y, en particular, con sus pacientes" (Fernández Álvarez et al., 2003, p. 118, la traducción me pertenece). Incluye no sólo el grado de involucración del terapeuta con sus pacientes, sino el lugar que su trabajo ocupa en el resto de su vida. Las polaridades de la misma oscilan entre baja y alta involucración. Los terapeutas con bajo nivel son aquellos que toman una actitud más distante, y que involucran pocos aspectos de su vida personal en el trabajo, a nivel de tiempo, relaciones familiares y personales, expectativas económicas y prestigio social en general; mientras que el otro extremo, está dado por terapeutas cuya actividad está mucho más implicada 
en su vida personal. La función de Involucración ha detectado asociaciones significativas con variables del terapeuta tales como: enfoque teórico (Casari et al., 2013), concepción epistemológica (Lee et al., 2013), entrenamiento profesional (Corbella et al., 2009 b) y personalidad (Genise, 2015).

Desde el inicio formal del constructo teórico en el año 1998 hasta la actualidad, pueden encontrarse más de 60 publicaciones diferentes, realizadas en diversos medios y sobre variadas temáticas (Casari, Albanesi, Maristany y Ison, 2016). Una de las líneas de investigación está relacionada al tipo de patología con la cual el profesional trabaja.

En la definición original del constructo EPT, se aclaraba que a pesar de su carácter estable el mismo podía modificarse en función de otros aspectos, de acuerdo con el tipo de paciente, patología y contexto en el cual se desarrollara la psicoterapia (Botella y Corbella, 2005). Es decir, existía una conjetura de que el EPT podía ser sensible a percibir diferencias estilísticas cuando se compararan terapeutas que trabajan con patologías específicas (y en instituciones especializadas). Entonces, desde el año 2006 se han publicado hasta el momento cinco investigaciones diferentes que se encargaron de estudiar el EPT en terapeutas de: neonatología (Vega, 2006), pacientes de severamente perturbados (Rial, Castañeiras, García, Gómez y Fernández-Álvarez, 2006), problemáticas relacionadas al maltrato infantil (Casari, Magni y Morelato, 2014), pacientes con enfermedades oncológicas (Gómez, 2015), y pacientes drogodependientes (Casari, Ison, Albanesi y Maristany, 2017).

En los estudios nombrados se realizó una metodología similar: se compararon dos o más grupos de terapeutas, donde uno estaba abocado al tratamiento de una patología específica (la mayoría de las veces pertenecían a instituciones especializadas), mientras que el segundo (o tercer) grupo eran psicólogos clínicos sin una dedicación específica a un tipo de patología a tratar. Si bien la cantidad de participantes a comparar no siempre fue exacta, sí se intentaba homologar los grupos en función de variables socio demográficas y profesionales (controlando igual proporción por sexo, edad, etc.). Luego se empleaba una prueba estadística para cumplir el objetivo de analizar la presencia de diferencias significativas en las funciones del EPT-C.

Rial et al. (2006) estudiaron el perfil de los terapeutas que trabajan con pacientes severamente perturbados (en adelante PSP), que provenían de centros psiquiátricos públicos y privados de la Ciudad de Buenos Aires y Mar del Plata, Argentina. Como resultado, los terapeutas del grupo en estudio se consideraron significativamente más distantes en la comunicación emocional con sus pacientes, con una menor involucración, y más pautados operativamente. También se indagó si al comparar terapeutas de distintas orientaciones teóricas pero que trabajaban en el mismo subgrupo (PSP o control), presentarían diferencias estadísticamente significativas en las funciones del EPT. Se encontraron tres resultados significativos, en las dimensiones Atencional, Expresiva y de Involucración en el subgrupo 
de profesionales dedicados a la atención de PSP $(n=30)$, donde en todos los casos los terapeutas cognitivo - integrativos $(\mathrm{n}=18)$ obtuvieron valores superiores a sus colegas psicoanalíticos $(\mathrm{n}=12)$. Esto implica que los mismos serían más focalizados en su atención, cercanos emocionalmente y con un mayor grado de compromiso.

En el mismo año, Vega (2006) publica su investigación con terapeutas de las Unidades de Cuidado Intensivo Neonatal (en adelante UCIN) de instituciones sanitarias de distintos lugares de Argentina. A nivel de resultados, se encontró una única diferencia significativa entre ambos subgrupos donde los terapeutas de las UCIN obtuvieron valores inferiores en la función Instruccional, lo cual implica mayor flexibilidad para establecer el encuadre de trabajo. No se encontraron diferencias significativas al comparar por enfoque teórico.

Casari et al. (2014) indagaron el EPT en profesionales que trabajaban en la atención de casos clínicos relacionados al maltrato infantil de la provincia de Mendoza, Argentina. En el análisis comparativo de diferencias de medias, se encontró que los psicólogos del grupo en estudio presentaron valores significativamente inferiores en la función Expresiva, lo cual implica mayor distancia en la comunicación emocional.

Un año después, Gómez (2015) indagó el perfil de terapeutas que trabajan en psico-oncología. Como resultado encontró diferencias significativas en las funciones Instruccional y Atencional, siendo inferiores. En el análisis por enfoque teórico, se encontraron diferencias con significación estadística entre las orientaciones en tres de las funciones terapéuticas: Atencional, Operativa y Expresiva.

Por último, Casari et al. (2017) indagaron el perfil del EPT en terapeutas que trabajaban en instituciones de atención en adicciones. Como resultado, se encontraron diferencias significativas en la función Expresiva e Instruccional, lo cual indica una mayor distancia emocional y un incremento en la rigidez del encuadre terapéutico en los terapeutas de pacientes drogodependientes.

Siguiendo en la línea del estudio del EPT en terapeutas que trabajan en diferentes poblaciones clínicas, una de las que no ha sido estudiada aún y podría presentar un estilo característico de trabajo, serían los terapeutas de niños con trastorno del espectro autista (TEA).

La conceptualización de TEA es reciente, debe su origen a la quinta edición del Manual Diagnóstico y Estadístico de los Trastornos Mentales (American Psychiatric Association [APA], 2014). Se empleó para reemplazar la antigua categoría de Trastornos Generalizados del Desarrollo, para referirse a un continuo que va desde cuadros leves a más graves, incluyendo el Autismo, el Síndrome de Asperger, el Trastorno Desintegrativo Infantil y el Trastorno Generalizado del Desarrollo no especificado (Serna, 2014).

El autismo es un trastorno complejo de naturaleza biológica del desarrollo infantil, con manifestaciones, causas y grados de gravedad muy variables. El mismo es un espectro de trastornos caracterizados por graves déficit del desarrollo, permanente y profundo. Afecta la socialización, la comunicación, la imagina- 
ción, la planificación y la reciprocidad emocional, y posee conductas repetitivas o inusuales. Los síntomas, por lo general, son la incapacidad de interacción social, el aislamiento y las estereotipias (Camino y López, 2006).

Si bien existen criterios comunes para el diagnóstico de TEA, parámetros como el nivel de inteligencia o el desarrollo del lenguaje influye en la amplia variación que puede encontrarse a nivel clínico (Fusar-Poli et al., 2017)

En relación a las dificultades en la comunicación, especialmente en el lenguaje expresivo (APA, 2014; Cadigan y Misal, 2007; Lane, Shepley y Lieberman-Betz, 2016), con las nuevas técnicas diagnósticas para la detección temprana de TEA, se ha podido estudiar el lenguaje de niños (Kim, Junker y Lord, 2014) encontrando que el desarrollo del mismo se relaciona con la disminución en los comportamientos restrictivos y repetitivos (Lane et al., 2016; Pry, Petersen y Baghdadli, 2005; RaySubramanian y Ellis Weismer, 2012), que es posible diseñar intervenciones para fortalecer el lenguaje expresivo espontáneo (Flippin y Watson, 2015; Lane, Lieberman-Betz y Gast, 2016), y que existen perfiles diferenciales en cuanto a la afectación del lenguaje (Ellawadia y Weismer, 2015; Ellis Weismer y Kover, 2015; Tek, Mesite, Fein y Naigles, 2014).

$\mathrm{Si}$ consideramos entonces que las dificultades en el lenguaje como modalidad de comunicación, y en las consecuencias que esto produce para mantener conversaciones con los demás (Naigles y Tek, 2017) la psicoterapia tradicional debe realizar ciertas adaptaciones para poder ser una alternativa terapéutica eficaz. Dado que la palabra se considera la herramienta central en la mayoría de los modelos psicoterapéuticos, la dificultad en esta área hace necesaria la búsqueda de modalidades de intervención diferentes a las tradicionales. Esto implica que entre los modos de intervención fundamentales el trabajo en equipo interdisciplinario se vuelva fundamental, con profesionales que integran diferentes áreas de evaluación, entre ellos médicos pediatras, psicólogos, psiquiatras e inclusive maestros integradores si es que el niño concurre a la escuela, siendo de principal importancia la participación de los padres de estos niños (Camino y López, 2006).

Por otro lado, la discapacidad intelectual implica la presencia de deficiencias en habilidades mentales generales que involucran el funcionamiento cognitivo y adaptativo (APA, 2014). Esta última y su comorbilidad con TEA puede ser estimada entre un 18 y 40 \% (Mbeya Mpaka et al., 2016; Tonnsen et al., 2016). Al manifestarse en conjunto, el desafío terapéutico es más complejo, porque suele estar asociado a elevados índice de comportamientos restrictivos y repetitivos (incluyendo autolesiones), y mayores dificultades a nivel comunicacional (Fusar-Poli et al., 2017; Oliver, Licencce y Richards, 2017; Tonnsen et al., 2016). También ocurre que cuando TEA cursa con una inteligencia normal, es más difícil encontrar herramientas diagnósticas válidas con amplio consenso en la comunidad científica (Baghdadli , Russet y Mottron, 2017).

Mulas et al. (2010) consideran que las intervenciones psicológicas en TEA son de tipo psicoeducativas, las cuales incluyen un amplio panorama de técnicas de 
intervención. En primer lugar, se encuentran las técnicas conductuales, las cuales se orientan a instruir al niño con TEA en ciertos comportamientos y habilidades utilizando técnicas como: Programa Lovaas, Análisis aplicado de la conducta (ABA), Pivotal Response Training(Entrenamiento de respuesta), Natural Language Paradigm, o Incidental Teaching (Sánchez, Martínez, Moriana, Luque y Alós, 2015). Luego estarían las intervenciones Evolutivas, donde se intenta que el niño desarrolle relaciones auténticas y demostrativas con las demás personas, a través de técnicas de comunicación, habilidades sociales y habilidades para la vida diaria (habilidades funcionales y motoras) en contextos paulatinos. Por último, están las intervenciones basadas en terapias, que promueven la mejora de habilidades comunicacionales, sociales y el avance sensoriomotor (terapia ocupacional), así como la integración de la familia en el tratamiento. (Salvadó, Palau, Clofent, Montero y Hernández, 2012).

Es importante destacar que el enfoque cognitivo conductual se manifiesta eficaz para beneficiar aspectos psicosociales y cognitivos de niños con autismo, siendo el más conocido el método ABA (Lang, Regester, Lauderdale, Ashbaugh y Haring, 2010). Este abordaje muestra efectividad a la hora de mejorar las habilidades sociales de personas con TEA (White, Ollendick, Scahill, Oswald y Albano, 2009).Existen condiciones generales que el terapeuta debe tener en cuenta antes de aplicar la terapia conductual e instruir la modificación de conductas en los niños con TEA: en primer lugar, se debe tener una evaluación completa del niño para conocer las necesidades a tratar; trabajar con el conocimiento de que cada caso es único, no se deben realizar comparaciones con otros casos, y tanto las necesidades, como los avances y progresos serán de cada caso en particular; no hay terapias universales, por lo tanto no habrá un programa diseñado que será efectivo en todos los caso de TEA que asistan a terapia; la planificación de actividades se realizarán en relación a las necesidades del niño y su familia; el terapeuta integrará a su trabajo a la familia y a la maestra si es que el niño concurre a la escuela, manteniendo una buena comunicación entre ellos; la constancia será clave para lograr adquirir nuevas habilidades (Garza, 2004).

La terapia cognitiva conductual también contiene objetivos generales los cuales no solo se aplican a la terapia, sino que también se los implementa a nivel educacional, es decir cuando el niño se encuentra integrado en una escuela. Ellos son: fortalecer la autonomía e independencia personal en niños con TEA, promover el autocontrol de determinadas conductas y ajuste al entorno, fortalecer habilidades sociales (capacidad de desarrollo en el ámbito habitual, comprensión de normas), ampliar estrategias de comunicación, y estimular procesos cognitivos básicos (pensamiento, atención y memoria).

Los últimos desarrollos acerca de los tratamientos para TEA proponen que la integración de diversos modos de intervención ha mostrado mejores resultados. Un ejemplo de esto es la NDBI (Naturalistic Developmental Behavioral Intervention) que conjuga la perspectiva conductual basada en la teoría del aprendizaje clásico 
(Pavlov, 2010), operante (Skinner, 1953) y por observación (Bandura, 1977) junto a los fundamentos de la psicología del desarrollo aportados por Piaget (1952), Bruner (1983), Vygotsky (1962) y otros. En donde se comprende que los reforzamientos sucesivos de la conducta obtienen mejores resultados si el niño tiene un papel activo en el aprendizaje y los padres pueden ejercer un rol de intermediarios en la conducta a aprender, especialmente cuando se trata del lenguaje. Las intervenciones tempranas desde esta perspectiva aplicada en los ambientes habituales en los que el niño o niña se desarrolla, han demostrado que la generalización de los aprendizajes es mucho mayor que en aquellos casos en que las intervenciones se realizan en contextos aislados como las instituciones o consultorios privados (Ingersoll, Meyer, Bonter, y Jelinek, 2012; Schreibman et al., 2015).Por estos motivos, es que los objetivos del presente artículo fueron:

- Comparar el perfil del EPT en dos grupos de psicólogos clínicos: uno dedicado al tratamiento de niños con TEA (en adelante Grupo TEA) y otro no especializado en una patología específica (en adelante Grupo Clínico).

- Analizar el posible efecto del enfoque teórico en cada grupo de trabajo.

\section{Metodología}

\section{Participantes}

La muestra quedó conformada por 64 psicólogos de diferentes provincias de Argentina: Mendoza, San Luis, Córdoba y Buenos Aires. Cada grupo estuvo conformado por igual cantidad de participantes: $n=32$. Las variables socio demográficas y socio profesionales que caracterizan a la muestra total se exponen a continuación (ver tabla 1).

Como puede observarse en las variables estudiadas la composición por grupo es similar. En su mayoría priman terapeutas del sexo femenino en ambos grupos $(78.1 \% / 75 \%)$, sin existir diferencias significativas por edad $(t(62)=.04, p=.96)$ o por años de experiencia profesional $(t(62)=.09, p=.92)$, así como también hay similar representación por enfoques teóricos en cada grupo.

En el Grupo TEA también se indagó la cantidad de horas semanales que se dedican al tratamiento de esta patología. El promedio fue de $15.56( \pm 8.46)$, oscilando desde 2 horas semanales a 35 horas. Los pacientes que atienden estos terapeutas son niños, cuyas edades oscilan desde $\operatorname{los} 4$ a 12 años. En las instituciones se dividen a los niños en diferentes salas, de acuerdo a capacidad intelectual y desarrollo del lenguaje (además del criterio por rango etario). El trabajo a nivel institucional, incluye constantemente la involucración de los padres desde el comienzo del tratamiento, realizando consultas, entrevistas, reuniones para padres y tallares mensuales, etc. Se les enseña a manejar los berrinches de los niños, cómo poner límites y se los informa constantemente sobre la evolución del proceso terapéutico. Con los niños se trabaja principalmente el desarrollo de habilidades sociales, motricidad, juego con los demás niños, manejo de pictogramas, entre otros. 
Tabla 1.

Variables socio profesionales del grupo clínico y grupo TEA ( $N=64)$

\begin{tabular}{crcc}
\hline & & Grupo Clínico (n= 32) & Grupo TEA (n= 32) \\
\hline \multirow{2}{*}{ Sexo } & Femenino & $78.1 \%$ & $75 \%$ \\
& Masculino & $21.9 \%$ & $25 \%$ \\
\hline \multirow{3}{*}{ Edad } & M (DE) & $32.5(7.7)$ & $32.65(6.04)$ \\
& Mínimo & 24 & 25 \\
& Máximo & 56 & 51 \\
& Rango & 32 & 26 \\
\hline \multirow{2}{*}{ Años de experiencia } & M (DE) & $5.47(5.7)$ & $5,53(5.6)$ \\
profesional & Mínimo & 0 & 0 \\
& Máximo & 27 & 27 \\
& Rango & 27 & 27 \\
\hline \multirow{2}{*}{ Enfoque teórico } & Cognitivo & & $25 \%$ \\
& Conductual & $25 \%$ & $40.6 \%$ \\
& Integrativo & $40.6 \%$ & $25 \%$ \\
& Psicoanalítico & $25 \%$ & $6.3 \%$ \\
& Sistémico & $6.3 \%$ & $3.1 \%$ \\
\hline
\end{tabular}

Nota. M (Media), DE (Desviación Estándar).

En cuanto al Grupo Clínico, los terapeutas trabajan principalmente con niños y adolescentes, y también adultos, con patologías relacionadas a trastornos de ansiedad y depresión, con formatos individuales de psicoterapia. Se desempeñan en diferentes contextos de trabajo: autónomo (independiente), empleados en el sector privado y público de salud. Los tratamientos que ejecutan en su mayoría son breves (hasta seis meses de duración) e intermedios (entre seis meses y dos años).

\section{Instrumentos}

Cuestionario de Estilo Personal del Terapeuta (EPT-C), versión abreviada (Castañeiras et al., 2008)

Este cuestionario, el cuál fue creado por Fernández Álvarez y García (1998), evalúa las cinco funciones del EPT: Atencional, Operativa, Instruccional, Involucración y Expresiva. Se utilizó la versión abreviada desarrollada por Castañeiras et al. (2008), compuesta por 21 ítems. El inventario expone como consigna investigar la forma en que el profesional realiza su trabajo, dando a conocer que no existen respuestas correctas y que debe ser espontáneo a la hora de establecer valores a los ítems. Los reactivos son exhibidos como afirmaciones a las que cada terapeuta debe manifestar su opinión en una escala tipo likert que va desde 1 (totalmente en desacuerdo), hasta 7 (totalmente de acuerdo). Las puntuaciones totales de las escalas del EPT se alcanzan por sumatoria simple de los ítems que componen cada una de ellas. La escala cuenta con adecuadas propiedades 
psicométricas tanto en validez (obtenidas en análisis factoriales exploratorios y confirmatorios) y confiabilidad (calculada a través del alfa de Cronbach).

\section{Encuesta semi-estructurada Ad Hoc.}

La misma se empleó para recabar información acerca de características de los terapeutas: sexo, edad, años de experiencia profesional, enfoque teórico y cantidad de horas semanales que trabaja en autismo (sólo en el Grupo TEA ésta última).

\section{Procedimiento}

En el Grupo TEA, se solicitó autorización a instituciones específicas de abordaje de esta patología explicando el propósito de la investigación a los respectivos directivos y coordinadores. Una vez obtenido el permiso, se contactó a profesionales psicólogos a los cuales se les comunicó de qué se trataba la investigación y se pasó a solicitar su colaboración voluntaria. Los profesionales firmaron un consentimiento informado donde se constataba que la información conseguida era confidencial, dejando constancia de su conformidad para participar de la investigación.

También se incluyó a terapeutas que trabajaban de manera particular en temáticas de TEA, contactando a los mismos a través de asociaciones de padres específicas o en sitios webs.

Los profesionales del Grupo Clínico fueron contactados en diversos medios tanto presenciales como virtuales. En ambos casos se dio a conocer el carácter confidencial de la prueba y el resguardo de los datos de identificación del profesional como también de los resultados individuales.

La recolección de datos fue realizada entre los meses de julio y noviembre del año 2016.

\section{Resultados}

Para cumplir el primer objetivo se aplicó una prueba $t$ para muestras independientes (ver tabla 2).

Tabla 2.

Comparación de funciones del EPT en ambos grupos $(n=64)$

\begin{tabular}{lccccc}
\hline Funciones EPT & Grupo TEA $(\mathbf{n}=\mathbf{3 2})$ & Grupo Clínico $(\mathbf{n = 3 2})$ & $\boldsymbol{t}$ & $\boldsymbol{p}$ & $\mathbf{d}$ \\
\cline { 2 - 6 } Atencional & $19.40(6.96)$ & $20.18(5.62)$ & -.49 & .62 & .12 \\
\hline Expresiva & $37.56(9.01)$ & $37.96(8.81)$ & -.18 & .85 & .04 \\
\cline { 2 - 6 } $\begin{array}{l}\text { Instruccional } \\
\text { Involucración }\end{array}$ & $29.37(9.36)$ & $31.64(6.65)$ & -1.12 & .26 & .27 \\
\cline { 2 - 6 } Operativa & $26.84(7.75)$ & $22.53(7.69)$ & 2.23 & .02 & .55 \\
\cline { 2 - 6 } & $25.62(8.74)$ & $23.85(7.17)$ & .88 & .38 & .22 \\
\hline
\end{tabular}

Como resultado se encontró una diferencia significativa en la función de 
Involucración $(t=2.23, p<.05)$, donde los terapeutas del Grupo TEA obtuvieron valores superiores, indicando un mayor grado de compromiso con la tarea y con los pacientes. El tamaño del efecto fue moderado $(\mathrm{d}=.55)$ (Cohen, 1988).

Para analizar si se producían diferencias significativas por enfoque teórico en las funciones del EPT al interior de cada grupo en estudio, se aplicó la prueba ANOVA de un factor. Para el análisis sólo se tomaron en consideración los tres grupos más representativos de la muestra: Integrativos $(\mathrm{n}=26)$, psicoanalíticos $(\mathrm{n}=$ 16) y cognitivos conductuales $(n=16)$ (ver tabla 3$)$.

Tabla 3.

Comparación de funciones del EPT entre ambos grupos, en relación a los enfoques teóricos $(n=58)$.

\begin{tabular}{|c|c|c|c|c|c|c|c|}
\hline & \multirow[b]{2}{*}{ Funciones EPT } & \multicolumn{3}{|c|}{ Grupo TEA } & \multicolumn{3}{|c|}{ Grupo Clínico } \\
\hline & & $M(D E)$ & $F$ & $p$ & $M(D E)$ & $F$ & $p$ \\
\hline \multirow{3}{*}{ Atencional } & Cognitivo Conductual & $27.12(5.24)$ & \multirow{3}{*}{12.92} & \multirow{3}{*}{.009} & $19,87(3.68)$ & \multirow{3}{*}{4.69} & \multirow{3}{*}{.01} \\
\hline & Psicoanalítico & $14(3.33)$ & & & $16(4.62)$ & & \\
\hline & Integrativo & $18.84(6.08)$ & & & $23(5.98)$ & & \\
\hline \multirow{3}{*}{ Expresiva } & Cognitivo Conductual & $36.12(5.08)$ & \multirow{3}{*}{4.19} & \multirow{3}{*}{.02} & $40.25(5.54)$ & \multirow{3}{*}{5.56} & \multirow{3}{*}{.01} \\
\hline & Psicoanalítico & $30.62(8.94)$ & & & $29.62(8.89)$ & & \\
\hline & Integrativo & $41.38(9.40)$ & & & $39.84(7.58)$ & & \\
\hline \multirow{3}{*}{ Instruccional } & Cognitivo Conductual & $35.5(8.15)$ & \multirow{3}{*}{2.05} & \multirow{3}{*}{.14} & $29.12(6.62)$ & \multirow{3}{*}{1.48} & \multirow{3}{*}{.17} \\
\hline & Psicoanalítico & $28.62(10.64)$ & & & $34.97(5.36)$ & & \\
\hline & Integrativo & $28.46(6.62)$ & & & $31.46(6.29)$ & & \\
\hline \multirow{3}{*}{ Involucración } & Cognitivo Conductual & $24.5(6.54)$ & \multirow{3}{*}{.68} & \multirow{3}{*}{.51} & $25(8.01)$ & \multirow{3}{*}{3.34} & \multirow{3}{*}{.05} \\
\hline & Psicoanalítico & $25.87(9.18)$ & & & $16.37(9.59)$ & & \\
\hline & Integrativo & $28.53(8.08)$ & & & $23.30(4.60)$ & & \\
\hline \multirow{3}{*}{ Operativa } & Cognitivo Conductual & $32.75(6.11)$ & \multirow{3}{*}{12.26} & \multirow{3}{*}{.008} & $24.87(6.17)$ & \multirow{3}{*}{3.00} & \multirow{3}{*}{.06} \\
\hline & Psicoanalítico & $16.37(3.88)$ & & & $18.80(6.60)$ & & \\
\hline & Integrativo & $27.53(8.35)$ & & & $26.38(7.66)$ & & \\
\hline
\end{tabular}

Como resultado, se encontraron las siguientes diferencias significativas. En la función Atencional se detectó una diferencia significativa tanto en el Grupo TEA como en el Grupo Clínico, en ambos casos los puntajes superiores los obtuvieron los terapeutas que adhieren al enfoque cognitivo conductual, y los valores inferiores los psicólogos psicoanalíticos. Esto implica mayor focalización atencional en la búsqueda de información terapéutica en el primer grupo de terapeutas.

En la función Expresiva se observó una diferencia significativa en ambos grupos, los mayores puntajes los obtuvieron los psicólogos de orientación integrativa del Grupo TEA; manifestando mayor cercanía emocional en el vínculo terapéutico. 
En correspondencia a esta función, los psicoanalistas presentan puntajes menores.

Por último, la función Operativa arrojó diferencias significativas sólo en el grupo TEA. Los valores superiores fueron obtenidos por los terapeutas cognitivoconductuales mostrando de esta forma inclinación por tratamientos más pautados; en el extremo opuesto estarían los psicoanalistas, con una inclinación por intervenciones espontaneas.

Seguidamente, se analizó si terapeutas de similar enfoque teórico pero pertenecientes a distintos grupos de trabajo podrían presentar diferencias significativas (ver tabla 4). Para ello se empleó la prueba no paramétrica U de Mann Whitney debido a que la distribución de las variables no fue normal, y también por el escaso número de participantes para el análisis $(\mathrm{n}<30)$. Como resultado se encontró que en el enfoque Cognitivo Conductual, los terapeutas del grupo TEA presentaron rangos de valores significativamente superiores en la función Instruccional y Operativa $(p<.05)$. Mientras que en el análisis de los terapeutas Integrativos, se encontró una única diferencia significativa donde los psicólogos especializados en TEA obtuvieron rangos de valores superiores $(p<.05)$

\section{Tabla 4}

\section{Comparación intragrupo según enfoque teórico}

\begin{tabular}{lcccccccc}
\cline { 2 - 10 } & \multicolumn{3}{c}{ Cognitivo Conductual } & \multicolumn{5}{l}{ Integrativos } \\
\cline { 2 - 10 } & $\begin{array}{c}\text { TEA } \\
(\mathbf{n}=\mathbf{8})\end{array}$ & $\begin{array}{c}\text { Clínico } \\
(\mathbf{n}=\mathbf{7})\end{array}$ & $\boldsymbol{U}$ & $\boldsymbol{p}$ & $\begin{array}{c}\text { TEA } \\
(\mathbf{n}=\mathbf{1 3})\end{array}$ & $\begin{array}{c}\text { Clínico } \\
(\mathbf{n}=13)\end{array}$ & $\boldsymbol{U}$ & $\boldsymbol{p}$ \\
\hline Atencional & 9,75 & 6 & 14 & .103 & 12,11 & 14,88 & 66,5 & .35 \\
\hline Expresiva & 7,18 & 8,92 & 21,5 & .448 & 15,8 & 11,19 & 54,5 & .12 \\
\hline Instruccional & 10,18 & 5,5 & 10,5 & .041 & 10,96 & 16,03 & 51,5 & .08 \\
\hline Involucración & 9,5 & 6,28 & 16 & .162 & 16,76 & 10,23 & 42 & .02 \\
\hline Operativa & 10,12 & 5,57 & 11 & .049 & 13 & 14 & 78 & .73 \\
\hline
\end{tabular}

Nota: Sólo se expresan los análisis que arrojaron resultados significativos en al menos una función del EPT, según el enfoque teórico del profesional.

\section{Discusión}

El primer objetivo específico, estuvo dirigido a analizar si existían diferencias en el EPT entre los terapeutas dedicados a los TEA y los que trabajan en psicología clínica general. Como resultado, se registró una única diferencia significativa en la función de Involucración, donde el Grupo TEA obtuvo mayor puntuación.

La función de Involucración hace referencia a las conductas explícitas e implícitas ligadas con el compromiso que domina el terapeuta en su actividad y con sus pacientes (Fernández Álvarez et al., 2003). Implica también el lugar que ocupa su trabajo como terapeuta en el contexto de su experiencia vital, evaluando de igual modo la disposición que tiene el terapeuta para ayudar a una persona que padece (Vides, Grazioso y García, 2012). Un alto nivel de involucración hace referencia a 
terapeutas que conectan muchos aspectos de su vida personal con la actividad profesional.

Si comparamos los resultados con las investigaciones realizadas en diferentes campos clínicos en función del EPT, sólo en una investigación se encontraron resultados significativos en la función Involucración. Rial et al. (2006), detectaron que el perfil de los terapeutas que trabajaban con PSP, incluía un bajo grado de involucración. Los autores concluían que podría tratarse de un mecanismo de protección de los terapeutas ante la elevada exigencia del trabajo clínico.

En relación a los resultados obtenidos en el presente estudio, se podría pensar que esto se encuentra relacionado a la rigurosidad que conlleva la tarea que ejercen los profesionales sobre los pacientes con TEA, ya que este es un trastorno complejo del desarrollo infantil, con diferentes orígenes y valores de gravedad (Camino y López, 2006).

Los niños con TEA necesitan de la atención de diversos profesionales desde su detección temprana y posiblemente a lo largo de toda su vida (De Iudicibus, 2011), por lo cual se podría pensar que los terapeutas deberían movilizarse en busca de recursos personales para lograr tratamientos efectivos en función a cada caso en particular, así como también capacitarse para lograr los cambios propuestos, ya que estos profesionales son los que poseen las herramientas necesarias y los estudios correspondientes para poder determinar esta patología y diseñar un modelo de abordaje (Camino y López, 2006). Castañeiras et al. (2008), afirmaban que los terapeutas para mejorar su aptitud profesional como instrumento terapéutico, debían formarse en las áreas pertinentes, para así ofrecer servicios de salud adecuados y seguros. Se podría pensar que de este modo el terapeuta presenta un gran compromiso con su trabajo y con sus pacientes, movilizándose en perfeccionarse para lograr resultados benéficos en estos niños.

La relación con dicha función también podría estar conectada al tratamiento que conlleva este trastorno, ya que el mismo debería realizarse de manera conjunta no sólo con otros profesionales (médicos, psiquiatras, docentes, etc.), sino sobre todo con los padres del niño con TEA (Camino y López, 2006), es por esto que los terapeutas deben ser abiertos y dispuestos, para así acompañar a la familia de manera correcta y alentarlos a buscar información si fuera necesario (Blenner, Reddy y Augustyn, 2011). Esto también estaría relacionado a un mayor grado de involucración, por su compromiso a la hora de realizar su trabajo.

Será necesario a lo largo del proceso mantener una relación afectiva entre terapeuta y niño, saber escuchar, comprender, entender que el ritmo de cada niño es diferente y explorar las interacciones que son primordiales para él (Kuras y Resnisky, 2011). Pareciera que el terapeuta de TEA tuviera que salir de su postura neutral para ir al encuentro otro ser humano cuya dificultad principal es vincularse, acortando de este modo las distancias y funcionando, quizás, según el concepto desarrollado por Vigotsky de zona de desarrollo proximal en cuanto a la socialización (Ruiz, 2015). 
Esta diferencia en la función de Involucración, también se vio reflejada al comparar a los terapeutas integrativos de ambos grupos de trabajo, siendo aun mayor el valor en los psicólogos del Grupo TEA. El análisis nos muestra a los terapeutas integrativos del Grupo TEA como aquellos con mayor compromiso con la tarea, diferenciándose de sus colegas psicoanalíticos del Grupo Clínico presentando menor grado de involucración.

Volviendo al análisis de las funciones del EPT, las demás dimensiones no manifestaron diferencias significativas, aunque sí se pudo observar que en el grupo TEA los valores encontrados arrojaron una inclinación a una atención menos abierta, intervenciones espontáneas, comunicación más distante y menor flexibilidad en el establecimiento de reglas para el encuadre.

En relación con el segundo y tercer objetivo, se examinó el enfoque teórico y su efecto sobre el EPT. Esta variable ha sido las más estudiada en las investigaciones referidas al EPT, pero son escasas las explicadas dadas para entender las diferencias encontradas (Casari, 2017). Estudios previos sobre el EPT en poblaciones clínicas, también habían encontrado diferencias significativas en los enfoques teóricos de cada grupo a comparar (Casari et al., 2014; Gómez, 2015; Rial et al., 2006).

En cuanto al segundo objetivo, las diferencias encontradas en el presente estudio se refirieron a las funciones Atencional, Expresiva y Operativa, donde sólo la última fue exclusiva del grupo TEA (las primeras dos también se replicaron en el Grupo Clínico). Mientras que en el tercer objetivo, las diferencias fueron en los terapeutas cognitivo conductuales, donde los psicólogos del Grupo TEA mostraron valores superiores en las funciones Instruccional y Operativa; en tanto que los psicólogos Integrativos obtuvieron puntajes mayores en la función de Involucración. Es decir, entre los últimos dos objetivos, todas las funciones del EPT se mostraron sensibles para percibir diferencias estilísticas según el enfoque teórico.

En la función Atencional, se obtuvieron puntajes superiores en los terapeutas de orientación cognitivo conductual e inferiores en los psicoanalíticos, coincidiendo con estudios previos (Vázquez y Gutiérrez, 2015). En investigaciones anteriores, se postuló al fenómeno de la atención flotante y a la asociación libre como técnicas propias del enfoque psicoanalítico (Schkolnik, 2008), que influyen en una actitud más receptiva en la función Atencional destacada en los terapeutas de dicha orientación teórica (Vázquez y Gutiérrez, 2015). Así como también al hecho opuesto, de que la atención sea más focalizada en los terapeutas cognitivoconductuales porque suelen ser terapias más breves y focalizadas sobre problemas específicos (Baringoltz, 2009). Los terapeutas cognitivos cognitivo conductuales del Grupo TEA son aún más focalizados que sus colegas del Grupo Clínico, lo cual podría ser lógico porque en éste último existen mayor cantidad de patologías a tratar entre sus participantes (principalmente trastornos de ansiedad y depresión). En el extremo opuesto estarían los psicólogos psicoanalistas de ambos grupos (TEA y Clínico), por lo que quizás aquí el enfoque teórico ejerza menor influencia que tratar a una patología específica en la función Atencional. 
Al analizar la función Expresiva, se observa que los profesionales más cercanos emocionalmente serían los integrativos que trabajan con TEA, por esto es que sería necesario a lo largo del proceso mantener una relación afectiva entre terapeuta y paciente con TEA, saber escuchar, comprender, entender que el ritmo de cada niño es diferente y explorar las interacciones que son primordiales para él (Kuras y Resnisky, 2011). Los psicoanalistas del grupo en estudio presentan valores inferiores, este resultado muestra coincidencias con investigaciones anteriores (Castañeiras et al., 2006, 2008). Se ha señalado desde el psicoanálisis, el énfasis en la mayor neutralidad posible de parte del terapeuta para no interferir con la transferencia del paciente, ya que a través de las proyecciones de este se expresa el síntoma a ser modificado y, para ello, el profesional de la psicología debe captar el modo de relacionarse del paciente, y no intentar estructurar la relación terapéutica (Virgilio, 2011). En el caso de esta función, es clara la identificación de enfoques teóricos (de ambos grupos) con las polaridades de la misma: mayor desarrollo (cercanía emocional) en Integrativos, y menor desarrollo (mayor distancia emocional) en Psicoanalistas; lo cual señala menor influencia de la variable tipo de patología sobre la función Expresiva.

La función Instruccional fue la menos sensible a percibir diferencias significativas, ya sea tanto en los análisis intragrupo del segundo objetivo, como también en la comparación intergrupo según enfoque teórico. Sólo en un caso se percibió una diferencia significativa: comparación entre terapeutas cognitivo conductuales del Grupo TEA en relación a sus pares del Grupo Clínico (mostrando mayor rigidez en los primeros). No hay un predominio claro de un grupo terapeutas que se identifique como aquel más flexible o rígido en el encuadro terapéutico. En estudios previos, si bien la función Instruccional ha sido señalada como quizás la más importante del EPT por sus vínculos con la alianza terapéutica (Corbella et al., 2007), ha sido también cuestionada por su escaso apoyo en los estudios psicométricos (Silva y Guedes, 2016). Por tal motivo debería continuar estudiándose, especialmente en el tratamiento de pacientes con TEA, porque la alianza terapéutica también desempeña un papel central (Brown et al., 2015; Goodman, Chung, Fischel yAthey-Lloyd, 2017; Klebanoff, 2015).

Por último, la función Operativa presenta puntajes altos en los profesionales del grupo TEA con enfoque cognitivo - conductual quienes señalan una inclinación por intervenciones más pautadas, y los psicólogos con enfoque psicoanalista del grupo TEA muestran puntajes bajos inclinándose de este modo a terapias espontaneas. Estos resultados también van en consonancia con investigaciones anteriores (Castañeiras et al., 2008). En el caso de los terapeutas cognitivo-conductuales, la tendencia a tratamientos pautados podría relacionarse al amplio repertorio de técnicas de intervención, las cuales hasta el momento serían las más utilizadas y eficaces (Mulas et al., 2010), presentando en sí un modelo a seguir a la hora de trabajar con ellas, siendo oportunas aplicarlas en edades tempranas para lograr resultados efectivos con el paso del tiempo (Salvadó et al., 2012). Mientras que en 
el caso de los profesionales que se definen como psicoanalistas, quizás se deba a una homogeneidad en el modo de abordaje técnico donde haya mayor conceptualización de la psicopatología subyacente, pero no tanta especificidad en divergencia de intervenciones técnicas tales como la interpretación y el análisis de la transferencia (Virgilio, 2011). Esta tendencia por intervenciones más pautadas, basadas en guías de tratamiento fue mayor inclusive en los terapeutas cognitivo conductuales del Grupo TEA al compararlos con sus colegas del Grupo Clínico; en tanto que sus colegas que adhieren al marco psicoanalítico están preferentemente inclinados por tratamientos no estructurados (en ambos grupos de trabajo).

Los resultados nos muestran a los terapeutas cognitivo-conductuales como aquellos más focalizados a nivel atencional y con preferencia por intervenciones pautados. Los psicólogos psicoanalistas tendrían un perfil opuesto al anterior: preferencia por una atención más abierta y por tratamientos espontáneos. Además, se caracterizarían por mayor distancia a nivel emocional. Finalmente, los terapeutas integrativos, poseerían mayor cercanía en la comunicación emocional, teniendo el perfil menos definido coincidiendo con estudios previos (Vázquez y Gutiérrez, 2015).

Para concluir, se mencionan las limitaciones de la presente investigación. La primera y más importante, es que debido al tipo de estudio planteado no se realiza una evaluación de resultado, no pudiendo inferir si el tipo de perfil es adecuado para el tratamiento. Podrían plantearse los siguientes interrogantes: ¿Es recomendable un grado alto o bajo de involucración? ¿qué consecuencias podrías traer a largo plazo una alta involucración?

Otra limitación que se presentó fue el criterio de inclusión de los profesionales. Quizás habiendo considerado condiciones más estrictas para participar (cierta cantidad de años de experiencia, nivel de capacitación), podrían haberse encontrado más diferencias en el perfil en estudio. Similares interrogantes fueron planteados en estudios de este tipo (Casari, 2017).

Sin embargo, estos resultados aportan nuevos datos acerca de que el EPT, una variable considerada relevante para el proceso y resultado en psicoterapia(Heinonnen, 2014), la cual es sensible a la hora de percibir diferencias según las poblaciones clínicas. Es evidente que los terapeutas no actúan de igual modo cuando trabajan con patologías específicas, por lo cual se remarca la necesidad de una formación más integral en TEA, brindando no sólo el conocimiento técnico a nivel de intervenciones, sino también las habilidades terapéuticas requeridas. 


\section{Referencias bibliográficas}

American Psychiatric Association (2014). Manual Diagnóstico y Estadístico de los Trastornos Mentales. DSMV. ( $5^{\circ}$ Ed.). Arlington: Médica Panamericana.

Baghdadli, A , Russet, F y Mottron, L. (2017). Measurement properties of screening and diagnostic tools for autism spectrum adults of mean normal intelligence: A systematic review. European Psychiatry, 44, 104-124. doi: 10.1016/j.eurpsy.2017.04.009

Bandura, A. (1977). Self-efficacy: toward a unifying theory of behavioral change. Psychological review, 84(2), 191-215. doi: 10.1037/0033-295X.84.2.191

Baringoltz, S. (2009). Integración de aportes cognitivos a la psicoterapia. Buenos Aires, Argentina: Paidós.

Blenner, S., Reddy, A, y Augustyn, M. (2011). Diagnóstico y tratamiento del autismo en la infancia. British Medical Journal, 343, 15-24. doi: 10.1136/bmj.d6238.

Botella, L., y Corbella, S. (2005). Neurobiología de la autorregulación afectiva, patrones de apego y compatibilidad en la relación terapeuta-paciente. Revista de psicoterapia, 61, 77-104. Recuperado de: http:// revistadepsicoterapia.com/neurobiologia-de-la-autorregulacion-afectiva-patrones-de-apego-y-compatibilidad-en-la-relacion-terapeuta-paciente.html

Brown, R., Iqbal, Z., Reynolds, L., Press, D. A., Shaker-Naeeni, H., Scrivener, L., ... y Murphy, S. (2015). Interrater reliability of treatment fidelity and therapeutic alliance measures for psychological therapies for anxiety in young people with autism spectrum disorders. International Journal of Developmental Disabilities, 61(4), 190-199. doi: 10.1179/2047387714Y.0000000050

Bruner, J. (1983). Child's talk: Learning to use language. Oxford, Reino Unido: Oxford University Press.

Cadigan, K., y Missall, K. N. (2007). Measuring expressive language growth in young children with autism spectrum disorders. Topics in Early Childhood Special Education, 27(2), 110-118. doi: 10.1177/ 02711214070270020101

Camino, L. y López, E. (2006). El espectro autista. Vox Pediátrica, 14(2), 7-15.

Casari, L. (2017). Estilo Personal del Terapeuta en profesionales que trabajan en el campo de las adicciones. Tesis de doctorado en Psicología (inédita). Facultad de Psicología, Universidad de San Luis. San Luis, Argentina.

Casari, L., Albanesi, S. y Maristany, M. (2013). Influencia del enfoque teórico en el Estilo Personal del Terapeuta. Psicogente, 29(16), 132-142.

Casari, L., Albanesi, S., Maristany, M. y Ison, M. (octubre, 2016). Estilo Personal del Terapeuta: Estado actual y perspectivas futuras. Presentación realizada en $2^{\circ}$ Congreso Latinoamericano para el Avance de la Ciencia Psicológica. Buenos Aires, Argentina.

Casari, L., Ison, M., Albanesi, S. y Maristany, M. (2017). Funciones del estilo personal del terapeuta en profesionales del campo de las adicciones. Pensamiento Psicológico, 15(1), 7-17. doi:10.11144/ Javerianacali.PPSI15-1.FEPT.

Casari, L., Magni, X. y Morelato, G. (octubre, 2014). Estilos Personal del Terapeuta en profesionales que trabajan en situaciones de maltrato infantil. Presentación realizada en $1^{\circ}$ Congreso Latinoamericano de Ciencia Psicológica. Buenos Aires, Argentina.

Castañeiras, C., García, F., Lo Bianco, J., y Fernández-Álvarez, H. (2006). Modulating effect of experience and theorical technical orientation on the Personal Style of the Therapist. Psychotherapy Research, 16(5), 587593.

Castañeiras, C., Ledesma, R., García, F. y Fernández-Álvarez, H. (2008). Evaluación del estilo personal del terapeuta: presentación de una versión abreviada del Cuestionario EPT-C. Terapia Psicológica, 26(1), 513.

Cohen, J. (1988). Statistical power analysis. Current directions in psychological science, 3(1), 98-101.

Corbella, S., Balmaña, N., Fernández-Álvarez, H., Saúl, L., Botella, L. y García, F. (2009 a). Estilo personal del terapeuta y teoría de la mente. Revista Argentina de Clínica Psicológica, 18(2), 125-133.

Corbella, S., Botella, L., Fernández-Álvarez, H., Saúl, L. y García, F. (2009 b). Developing the Therapist's Personal Style. Presentación realizada en $40^{\text {th }}$ International Meeting of the Society for Psychotherapy Research. Santiago de Chile, Chile.

Corbella, S., Fernández-Álvarez, H., Botella, L., García, F., Lo Bianco, J. y Canizzarro, A. (2007 a). Compatibility between Therapist's Personal Style and Patient's Personality. Presentación realizada en $38^{\text {th }}$ Annual Meeting of Society for Psychotherapy Research. Wisconsin, Estados Unidos.

De Iudicibus, L (2011). Trastornos Generalizados del desarrollo. Revista Argentina de Clínica Neuropsiquiatrica, $17(1), 5-41$. 
Ellawadi, A. B., y Weismer, S. E. (2015). Using spoken language benchmarks to characterize the expressive language skills of young children with Autism Spectrum Disorders. American journal of speech-language pathology, 24(4), 696-707. doi:10.1044/2015 AJSLP-14-0190

Ellis Weismer, S., y Kover, S. T. (2015). Preschool language variation, growth, and predictors in children on the autism spectrum. Journal of ChildPsychology and Psychiatry, 56(12), 1327-1337. DOI: 10.1111/jcpp.12406

Fernández-Álvarez, H. y García, F. (1998). El estilo personal del terapeuta: Inventario para su evaluación. En S. Gril, A. Ibañez, I. Mosca y P.L.R. Sousa (Eds.), Investigación en Psicoterapia (pp. 76-84). Pelotas: Educat.

Fernández-Álvarez, H., García, F. y Schreb, E. (1998). The research program at AIGLE. Journal of Clinical Psychology, 54(3), 343-359. doi: 10.1002/(SICI)1097-4679(199804)54:3<343::AID-JCLP5>3.0.CO;2-Q.

Fernández-Álvarez, H., García, F., Lo Bianco, J., y Corbella, S. (2003). Assessment questionnaire on the personal style of the therapist PST-Q. Clinical Psychology y Psychotherapy, 10, 116-125. doi: 10.1002/cpp.358.

Flippin, M., y Watson, L. R. (2015). Fathers' and Mothers' Verbal Responsiveness and the Language Skills of Young Children with Autism Spectrum Disorder. American Journal of Speech-Language Pathology, 24(3), 400-410. doi:10.1044/2015 AJSLP-13-0138

Fusar-Poli, L., Brondino, N., Orsi, P., Provenzani, U., De Micheli, A., di Nemi, S. U., ... y Politi, P. (2017). Longterm outcome of a cohort of adults with autism and intellectual disability: A pilot prospective study. Research in developmental disabilities, 60, 223-231. doi: 10.1016/j.ridd.2016.10.014

Garza, J. (2004) Manual para padres de niños autistas. Bogotá: Psicom editores.

Genise, G. (2015). Relación entre el estilo personal del terapeuta, estilo de apego y factores de personalidad del terapeuta. Psicodebate, 15(1), 9-22.

Genise, S. y García, F. (octubre, 2016). Estudio de correlación entre el EPT, estilo de apego adulto y factores de personalidad. Presentación realizada en $2^{\circ}$ Congreso Latinoamericano para el Avance de la Ciencia Psicológica. Ciudad Autónoma de Buenos Aires, Argentina.

Gómez, B. (2015). El Estilo Personal del Terapeuta en psico-oncología. Tesis doctoral de Psicología (inédita). Universidad del Salvador, Argentina.

Goodman, G., Chung, H., Fischel, L., y Athey-Lloyd, L. (2017). Simulation modeling analysis of sequential relations among therapeutic alliance, symptoms, and adherence to child-centered play therapy between a child with autism spectrum disorder and two therapists. Clinical Child Psychology and Psychiatry, 22(3), 1-12. DOI: $10.1177 / 1359104517691082$

Grzona, S., Muñoz, M., Casari, L. y Videla, R. (octubre, 2016). Estilo Personal del Terapeuta en estudiantes. Comparación pre-post prácticas clínicas. Presentación realizada en $2^{\circ}$ Congreso Latinoamericano para el Avance de la Ciencia Psicológica. Buenos Aires, Argentina.

Heinonen, E. (2014). Therapists' professional and personal characteristics as predictors of working alliance and outcome in psychotherapy. Accademic Dissertation. Research/National Institute for Health and Welfare.

Hermosa-Bosano, C. (2010). Influencia de los Rasgos de Personalidad y el Estilo Personal del Terapeuta Aprendiz sobre las Percepciones de la Alianza Terapéutica. Tesis de grado de Lic. en Psicología Clínica (inédita). Colegio de Artes Liberales, Universidad de San Francisco de Quito, Perú.

Ingersoll, B., Meyer, K., Bonter, N., y Jelinek, S. (2012). A comparison of developmental, social-pragmatic and naturalistic behavioral interventions on language use and social engagement in children with autism. Journal of Speech, Language, and Hearing Research, 55(5), 1301-1313. DOI: 10.1044/1092-4388(2012/10-0345).

Kim, S. H., Junker, D., y Lord, C. (2014). Observation of spontaneous expressive language (OSEL): a new measure for spontaneous and expressive language of children with autism spectrum disorders and other communication disorders. Journal of autism and developmental disorders, 44(12), 3230-3244. doi:10.1007/s10803-0142180-0

Klebanoff, S. (2015). The therapeutic alliance in cognitive-behavioral therapy for children with autism and anxiety. Tesis doctoral (inédita). University of California, Los Angeles.

Kuras, S. y Resnisky, S. (2011). El acompañamiento terapéutico como dispositivo. Buenos Aires: Letra Viva.

Lane, J. D., Lieberman-Betz, R., y Gast, D. L. (2016). An analysis of naturalistic interventions for increasing spontaneous expressive language in children with autism spectrum disorder. The Journal of Special Education, 50(1), 49-61. DOI: 10.1177/0022466915614837

Lane, J. D., Shepley, C., y Lieberman-Betz, R. (2016). Promoting Expressive Language in Young Children with or At-Risk for Autism Spectrum Disorder in a Preschool Classroom. Journal of autism and developmental disorders, 46(10), 3216-3231. doi: 10.1007/s10803-016-2917-z

Lang, R., Regester, A., Lauderdale, S., Ashbaugh, K. y Haring, A. (2010). Treatment of anxiety in autism spectrum disorders using cognitive behaviour therapy: A systematic review. Developmental Neurorehabilitation, 13, 53-63. doi: 10.3109/17518420903236288 
Lee, J. A., Neimeyer, G. J. y Rice, K. G. (2013). The Relationship between Therapist Epistemology, Therapy Style, Working Alliance, and Interventions Use. American Journal of Psychotherapy, 67(4), 323-345.

Moya, L.(2015). Influencia del Estilo Personal del Terapeuta en la calidad de la alianza. Tesis de Lic. en Psicología (inédita). Pontificia Universida de Comillas de Madrid.

Mpaka, D. M., Okitundu, D. L. E. A., Ndjukendi, A. O., N'situ, A. M., Kinsala, S. Y., Mukau, J. E., ... y Steyaert, J. (2016). Prevalence and comorbidities of autism among children referred to the outpatient clinics for neurodevelopmental disorders. The Pan African Medical Journal, 25, 1-10. doi: 10.11604/ pamj.2016.25.82.4151

Mulas, F., Ros-Cervera, G., Millá, M. G., Etchepareborda, M. C., Abad, L., y Téllez de Meneses, M. (2010). Modelos de intervención en niños con autismo. Revista Neurológica, 50(3), 77-84.

Naigles, L. R., y Tek, S. (2017). Form is easy, meaning is hard'revisited:(re) characterizing the strengths and weaknesses of language in children with autism spectrum disorder. WIREs Cognitive Science, 8(4), 14-38. doi: $10.1002 /$ wcs. 1438

Oliver, C., Licence, L., y Richards, C. (2017). Self-injurious behaviour in people with intellectual disability and autism spectrum disorder. Current opinion in psychiatry, 30(2), 97-101. doi: 10.1097/ YCO.0000000000000313

Pavlov, P. I. (2010). Conditioned reflexes: an investigation of the physiological activity of the cerebral cortex. Annals of neurosciences, 17(3), 136-141. doi: 10.5214/ans.0972-7531.1017309

Piaget, J. (1952). The origins of intelligence in children. New York: International Universities Press.

Pry, R., Petersen, A., y Baghdadli, A. (2005). The relationship between expressive language level and psychological development in children with autism 5 years of age. Autism, 9(2), 179-189. DOI: 10.1177/1362361305047222

Ray-Subramanian, C. E., y Weismer, S. E. (2012). Receptive and expressive language as predictors of restricted and repetitive behaviors in young children with autism spectrum disorders. Journal of autism and developmental disorders, 42(10), 2113-2120. DOI 10.1007/s10803-012-1463-6

Rial, V., Castañeiras, C., García, F., Gómez, B. y Fernández-Álvarez, H. (2006). Estilo personal de terapeutas que trabajan con pacientes severamente perturbados: un estudio cuantitativo y cualitativo. Revista de la Asociación Española de Neuropsiquiatría, 26(98), 191-208.

Ruiz, C. (2015). Hacia una comprobación experimental de la zona de desarrollo próximo de Vigotsky. Ciencia ergo sum, 22(2), 167-171.

Salvadó, B., Palau, M., Clofent, M, Montero, M. y Hernández, M. (2012) Modelos de intervención global en personas con trastorno del espectro autista. Revista de Neurología, 54(1), 63-71.

Sánchez, M., Martínez, E., Moriana, J., Luque, B. y Alós, F. (2015) La atención temprana en los trastornos del espectro autista. Psicología Educativa, 21(1), 55-63.

Schkolnik, F. (2008). Práctica psicoanalítica: Trabajando las diferencias. ¿Una práctica psicoanalítica o varias? Revista Uruguaya de Psicoanálisis, 106, 7-21.

Schreibman, L., Dawson, G., Stahmer, A. C., Landa, R., Rogers, S. J., McGee, G. G., ... y McNerney, E. (2015). Naturalistic developmental behavioral interventions: Empirically validated treatments for autism spectrum disorder. Journal of Autism and Developmental Disorders, 45(8), 2411-2428. DOI 10.1007/s10803-0152407-8.

Serna, S. (2014). Evaluación de la Teoría de la Mente en niños diagnosticados dentro del espectro Autista. Tesis de Lic. en Psicología (inédita). Facultad de Educación y Trabajo Social, Universidad de Valladolid. Valladolid, España.

Silva, E. y Guedes, S. (2016). Questionário Reduzido do Estilo Pessoal do Terapeuta: evidências de validade. Avaliçãao Psicológica, 15(3), 298-307. DOI: 10.15689/ap.2016.1503.02

Skinner, B E (1953). Science and human behavior. Nueva York: Macmillan

Tek, S., Mesite, L., Fein, D., y Naigles, L. (2014). Longitudinal analyses of expressive language development reveal two distinct language profiles among young children with autism spectrum disorders. Journal of autism and developmental disorders, 44(1), 75-89. doi: 10.1007/s10803-013-1853-4

Tonnsen, B. L., Boan, A. D., Bradley, C. C., Charles, J., Cohen, A., y Carpenter, L. A. (2016). Prevalence of Autism Spectrum Disorders among Children with Intellectual Disability. American journal on intellectual and developmental disabilities, 121(6), 487-500. doi: 10.1352/1944-7558-121.6.487

Vázquez, L. y Gutiérrez, M. (2015). Orientación teórico-técnica y EPT. Revista Argentina de Clínica Psicológica, 24(2), 133-142.

Vega, E. (2006). El psicoterapeuta en neonatología. Rol y estilo personal. Bs. As.: Lugar editorial.

Vides, A., Grazioso, M. y García, C. (2012). El Estilo Personal del Terapeuta Guatemalteco en el interior del país. Revista Argentina de Clínica Psicológica, 21(1), 33-43. 
Virgilio, A. (2011). Modelo dinámico de la terapia. En H. Fernández Álvarez (Comp.) Paisajes de la psicoterapia: Modelos, aplicaciones y procedimientos (pp. 99 - 132). Buenos Aires, Argentina: Polemos.

Vygotsky, L. S. (1962). Thought and language. Cambridge, MA: MIT press.

White, S.W., Ollendick, T., Scahill, L., Oswald, D., y Albano, A.M. (2009). Preliminary efficacy of a cognitivebehavioral treatment program for anxious youth with autism spectrum disorders. Journal of Autism and Developmental Disorders, 39, 1652-1662. Doi: 10.1007/s10803-009-0801-9 